TITLE:

\title{
Structure of the breakpoint region on current-voltage characteristics of intrinsic Josephson junctions
}

\section{$\operatorname{AUTHOR(S):~}$}

Shukrinov, Yu M.; Mahfouzi, F.; Suzuki, M.

\section{CITATION:}

Shukrinov, Yu M.... [et al]. Structure of the breakpoint region on current-voltage

characteristics of intrinsic Josephson junctions. PHYSICAL REVIEW B 2008, 78(13): 134521.

ISSUE DATE:

2008-10

URL:

http://hdl.handle.net/2433/84616

RIGHT:

(c) 2008 The American Physical Society 


\title{
Structure of the breakpoint region on current-voltage characteristics of intrinsic Josephson junctions
}

\author{
Yu. M. Shukrinov, ${ }^{1,2}$ F. Mahfouzi, ${ }^{1}$ and M. Suzuki ${ }^{2,3}$ \\ ${ }^{1}$ BLTP, JINR, Dubna, Moscow Region 141980, Russia \\ ${ }^{2}$ Photonics and Electronics Science and Engineering Center, Kyoto University, Kyoto 615-8510, Japan \\ ${ }^{3}$ Department of Electronic Science and Engineering, Kyoto University, Kyoto 615-8510, Japan
}

(Received 12 September 2008; published 16 October 2008)

\begin{abstract}
A fine structure of the breakpoint region in the current-voltage characteristics of the coupled intrinsic Josephson junctions in the layered superconductors is found. We establish a correspondence between the features in the current-voltage characteristics and the character of the charge oscillations in superconducting layers in the stack and explain the origin of the breakpoint region structure.
\end{abstract}

DOI: $10.1103 /$ PhysRevB.78.134521

PACS number(s): 74.50.+r, 74.25.Fy, 74.81.Fa, 74.20.De

A system of Josephson junctions attracts a great interest from both scientific and practical sides. To describe it, a system of coupled nonlinear equations is used, which might be solved numerically only. Recently observed ${ }^{1}$ powerful enough coherent radiation from the stack of the intrinsic Josephson junctions (IJJ) in layered superconductor $\mathrm{Bi}_{2} \mathrm{Sr}_{2} \mathrm{CaCu}_{2} \mathrm{O}_{8}$ (BSCCO) opens possibilities for different applications.

In Refs. 2-4 we studied the multiple branch structure of the current-voltage characteristics (CVC) of IJJ and showed that the branches have a breakpoint (BP) and a breakpoint region (BPR) before transition to another branch. The BP current is determined by the creation of the longitudinal plasma waves (LPW) with a definite wave number $k$, which depends on the coupling parameter $\alpha$, the dissipation parameter $\beta$, the number of junctions in the stack, and the boundary conditions. We generalized the McCumber-Stewards dependence of the return current for the case of IJJ in the HTSC, and the oscillation of the breakpoint current as a function of parameters $\alpha$ and $\beta$ was found. Based on the idea of the parametric resonance in the stack of IJJ, we explained this oscillation as a result of the creation of longitudinal plasma waves with different wave numbers. A good qualitative agreement of the results of modeling of the $\alpha \beta$ dependence of the BP current with the results of simulation was obtained. We demonstrated that the $\alpha \beta$ dependence of the BP current is an instrument to determine the mode of LPW created at the breakpoint in the stacks with a different number of junctions. So, the breakpoint in the CVC was explained, but the nature and features of the breakpoint region were not clear at that time.

In this paper we study theoretically the phase dynamics of a stack of IJJ in the high- $T_{c}$ superconductors. The CVC of IJJ are numerically calculated in the framework of capacitively coupled Josephson-junction model with diffusion current. ${ }^{5,6}$ From the results of the numerical simulations we predict a fine structure of the breakpoint region in the CVC. A correspondence between the features in the CVC and the character of the charge oscillations on different superconducting layers is established and the origin of the breakpoint region structure is explained. In the BPR the plasma mode is a stable solution of the system and this fact might be used in some applications, particularly, in high-frequency devices such as terahertz oscillators and mixers. We consider that the breakpoint phenomenon might be used to understand the mechanism of the powerful coherent radiation from the stack of the IJJ in BSCCO.

To find the CVC of the stack of IJJ, we solve a system of dynamical equations for the gauge-invariant phase differences $\varphi_{l}(\tau)=\theta_{l+1}(\tau)-\theta_{l}(\tau)-\frac{2 e}{\hbar} \int_{l}^{l+1} d z A_{z}(z, \tau)$ between superconducting layers ( $S$ layers) for the stacks with a different number of IJJ in the framework of the capacitively coupled Josephson-junction model with diffusion current (CCJJ + DC model $),{ }^{5,6}$ where $\theta_{l}$ is the phase of the order parameter in the $S$-layer $l$ and $A_{z}$ is the vector potential in the barrier. We use a dimensionless time $\tau=t \omega_{p}$, where $\omega_{p}$ is the plasma frequency $\omega_{p}=\sqrt{2 e I_{c} / \hbar C}, I_{c}$ is the critical current, and $C$ is the capacitance. The system of equations has a form $\partial^{2} \varphi_{l} / \partial \tau^{2}=\Sigma_{l^{\prime}} A_{l l^{\prime}}\left[I-\sin \varphi_{l^{\prime}}-\beta \partial \varphi_{l^{\prime}} / \partial \tau\right]$ with matrix A given in Ref. 3 for periodic and nonperiodic boundary conditions (BC). In our simulations we measure the voltage in units of $V_{0}=\hbar \omega_{p} /(2 e)$ and the current in units of the $I_{c}$. The CVC and time dependence of the charge oscillation in the $S$ layers are simulated at $\alpha=1$ and $\beta=0.2$ and periodic BC. The details concerning the model and numerical procedure are presented in Refs. 3, 4, and 6 In the inset to Fig. 1(a) we show the result of simulation of the CVC for a stack with nine IJJ. It demonstrates an equidistant multiple branch structure in the hysteresis region and the breakpoint location. The transition from the state where the outermost branch with all junctions is in a rotating state $(R \text { state })^{7}$ to a state with some junctions in an oscillating state ( $O$ state) happens in a resonance region where the LPW with a definite wave number is created. In the stack with 11 IJJ with mentioned above values of $\alpha=1$ and $\beta=0.2$, the LPW with $k=10 \pi / 11 d$ is created, where $d$ is the lattice period along the $c$ axis. ${ }^{3,4}$ In Fig. 1(a) we can see that the CVC exhibits a fine structure in the BPR. The BPR has different parts with a different dependence of the voltage on the bias current. For a given stack we can see clear five parts in the CVC separated by some points $T, Y, C_{1}$, and $C_{2}$ where the character of the $\mathrm{CVC}$ is changed. The letters $B$ and $J$ show the breakpoint and jumping point, respectively, to the other branch. Points $S$ on the CVC will be discussed later.

To find the origin of this structure and explain its features, we study time dependence of the charge in the superconducting layers. Using Maxwell's equation $\operatorname{div}\left(\varepsilon \varepsilon_{0} E\right)=\rho$, where $\varepsilon$ 

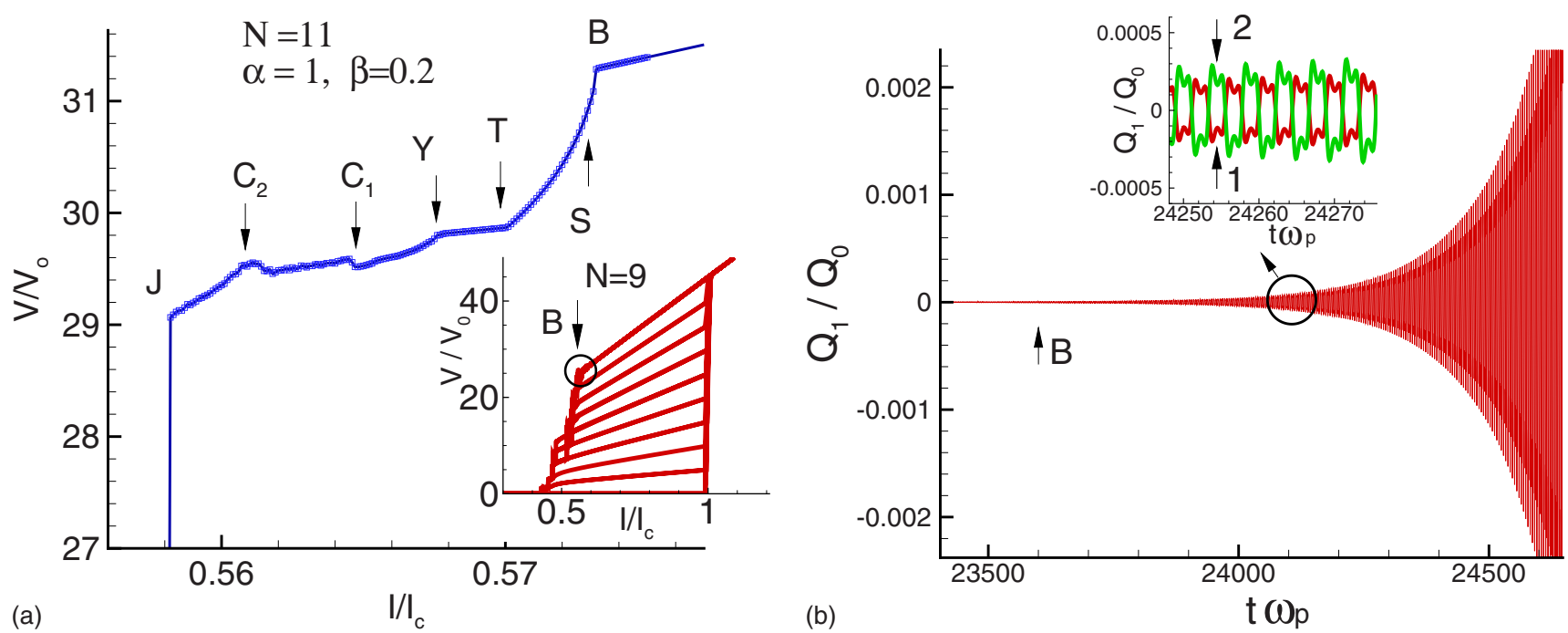

FIG. 1. (Color online) (a) The fine structure of the BPR in the outermost branch in the CVC of the stack with 11 IJJ. The insert shows a total branch structure in the CVC of the stack with nine IJJ and the BP location. (b) Charge oscillation in the first layer in the beginning of $B-S$ part of the BPR. The inset shows the oscillations in layers 1 and 2 .

and $\varepsilon_{0}$ are relative dielectric and electric constants, respectively, we express the charge density $Q_{l}$ (we call it just charge) in the $S$-layer $l$ by the voltages $V_{l}$ and $V_{l+1}$ in the neighbor insulating layers $Q_{l}=Q_{0} \alpha\left(V_{l+1}-V_{l}\right)$, where $Q_{0}$ $=\varepsilon \varepsilon_{0} V_{0} / r_{D}^{2}$ and $r_{D}$ is Debay screening length. Solution of the system of dynamical equations for the gauge-invariant phase differences between $S$ layers gives us the voltages $V_{l}$ in all junctions in the stack, and it allows us to investigate the time dependence of the charge on each $S$ layer.

The "time dependence" actually consists of time and bias current variation. We solve the system of dynamical equations for phase differences at fixed value of bias current $I$ in some time interval $\left(0, T_{m}\right)$ of dimensionless time $\tau=t \omega_{p}$ with time step $\delta \tau$, where $t$ is real time. This interval is used for time averaging procedure. Then we change the bias current by $\delta I$ and repeat the same procedure for the current $I+\delta I$ in new time interval $\left(T_{m}, 2 T_{m}\right)$. The values of the phase and derivative of phase in the end of the first time interval are used as initial conditions for second time interval and so on. In our simulations we put $T_{m}=250, \delta \tau=0.05$, and $\delta I=0.0001$, and total recorded time was calculated as $\tau+T_{m}\left(I_{0}-I\right) / \delta I$, where $I_{0}$ is an initial value of the bias current for time-dependence recording.

In the Fig. 2 the profile of time dependence of the charge in the $S$ layers in the stack with nine IJJ is combined with the CVC of the outermost branch at $\alpha=1, \beta=0.2$, and periodic BC. We show oscillations in the (a) first, (b) third, and (c) fourth layers only because in the other layers its character close to the behavior in these layers. To avoid large data files in the procedure of time-dependence recording and to present the CVC and time dependence with the same precision, we have limited the averaging time interval by $T_{m}$ $=250$. It leads to a more smeared CVC in this figure than the one shown in Fig. 1(a) $\left(T_{m}=25000\right)$; but the main features of the CVC are preserved. Moreover, such an averaging also allows us to find one more characteristic point $S$ within the BPR.

We have investigated the influence of the noise in bias current on the CVC, which is generated arbitrary in each IJJs. The noise is produced by random number generator and its amplitude is normalized to the critical current value $I_{c}$. Figure 3 presents the CVC of the outermost branch in the vicinity of the breakpoint for three values of noise amplitude: $10^{-6}, 10^{-8}$, and $10^{-13}$ of a stack with $11 \mathrm{IJJ}$. We see that the first four parts of the BPR from the point $B$ till the point $C_{1}$ are not affected by noise in current practically. But its strong effect has a place in the $C_{1}-J$ region. The noise in bias current smears the CVC essentially here because the four first parts of the BPR demonstrate a behavior close to the regular one, but the part $C_{1}-J$ shows the chaotic behavior. The voltage value is very sensitive to the small changes in the value of current here.

As we can see in the Fig. 2, the features of the CVC are in correlation with the features of time dependence of the charge on the $S$ layer. This fact can be explained by the idea of the parametric resonance at point $B$. As was shown in Ref. 8 , the system of equations for CCJJ has a solution corresponding to the LPW propagating along the $c$ axis. A frequency of the LPW at $I=0$ and $\beta=0$ is $\omega_{\mathrm{LPW}}(k)$ $=\omega_{p} \sqrt{1+2 \alpha(1-\cos k d)}$, where $k$ is wave vector of the LPW. At point $B$ the Josephson oscillations excite the LPW by their periodical actions. The frequency of Josephson oscillations is determined by the voltage value in the junction; so at $\omega_{J}=2 \omega_{\mathrm{LPW}}$ the parametric resonance is realized and the LPW is created.

The sharp increase in the oscillation amplitude in the first layer of the stack with nine IJJ in the beginning of the $B-S$ range is demonstrated in Fig. 1(b), where the LPW with wave vector $k=8 \pi / 9 d$ is created. This mode is close to the $\pi$ mode $(k=\pi / d)$. In the $\pi$ mode charges on the nearest lattice points oscillate in the opposite directions and the inset showed by numbers 1 and 2 that the charges oscillate in layers 1 and 2 in opposite directions too. But the amplitude of oscillations in layer 2 is smaller than in layer 1, which demonstrate the difference of this mode from the $\pi$ mode.

Fast Fourier transformation (FFT) analysis for the time 

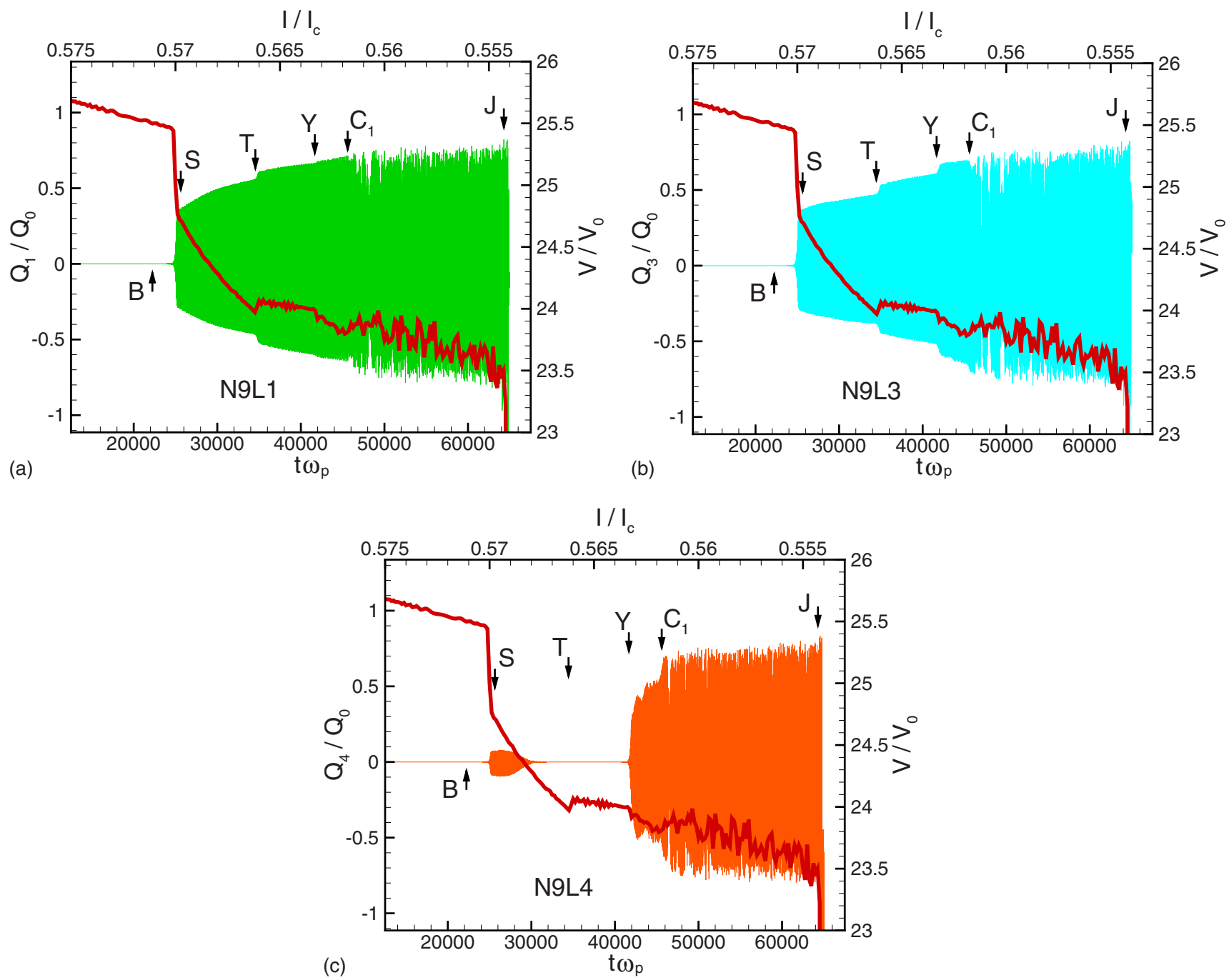

FIG. 2. (Color online) (a) Profile of time dependence of the charge in the $S$-layers in the stack with nine IJJ: (a) In the first layer. (b) In the third layer. (c) In the fourth layer. The red curve shows the CVC of this stack in the BPR.

dependence of the charge in third $S$ layer is presented in Fig. 4. The time domain samples for FFT are collected across the interval $\left(0, T_{m}\right)$ at each step of the bias current. Figure 4(a) shows that before the $\mathrm{BP}$ at $I / I_{c}=0.573$ the Josephson frequency $\omega_{J}=0.4542 \times 2 \pi \omega_{p}=2.8538 \omega_{p}$ is observed only. In accordance with the parametric resonance, we can see the peak at $\omega=0.2246 \times 2 \pi \omega_{p}=1.4112 \omega_{p}$ corresponding to the LPW frequency $\omega_{\text {LPW }}$ in the $B-S$ region [Fig. 4(b)]. We observe also the peak at $\omega=0.6738 \times 2 \pi \omega_{p}=4.2336$ corresponding to the sum of the Josephson and LPW frequencies $\omega_{J}+\omega_{\mathrm{LPW}}$. A sharp increase in the oscillation amplitude at resonance point is observed on other layers in the $B-S$ region also. The oscillation in the $S-T$ part is similar to those for all layers in the stack excluding layer 4. Because we consider the periodic $\mathrm{BC}$ along $c$ axis here, the layer number is used just for distinctness. As we can see in Fig. 2(c), the amplitude of the charge oscillation in layer 4 in the $B-Y$ region is essentially smaller than that in the other layers. The FFT analysis of the $S-T$ part shows the same peaks as for $B-S$ part, but here we can see an additional peak 0.4395 $\times 2 \pi \omega_{p}=2.7615 \omega_{p}$, which value approximately equal to $2 \omega_{\mathrm{LPW}}$. As it is demonstrated in Fig. 5(b), the character of the

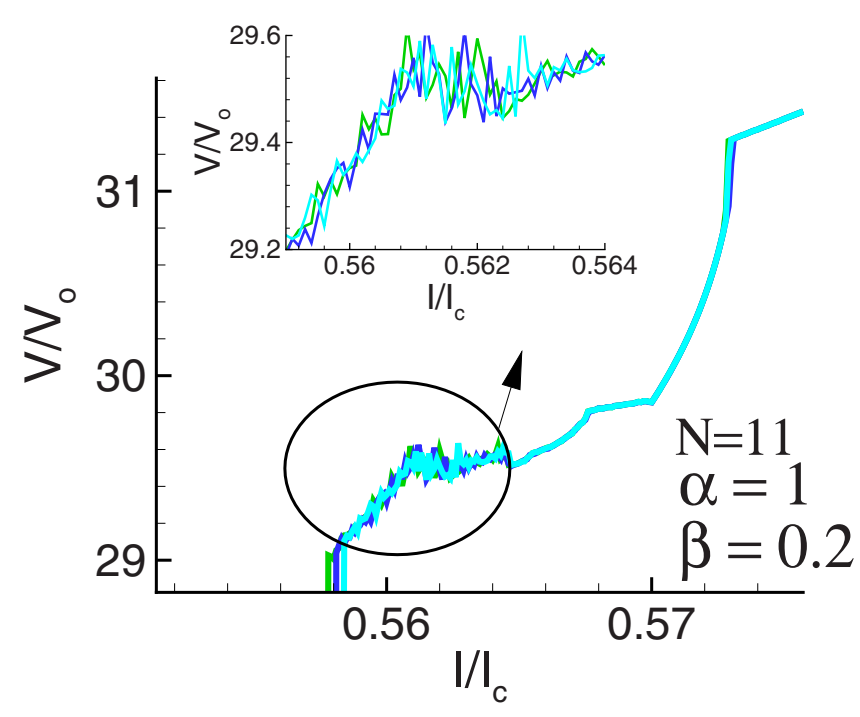

FIG. 3. (Color online) Superposition of the three CVC with the amplitudes of noise in the current $10^{-6}, 10^{-8}$, and $10^{-13}$. 

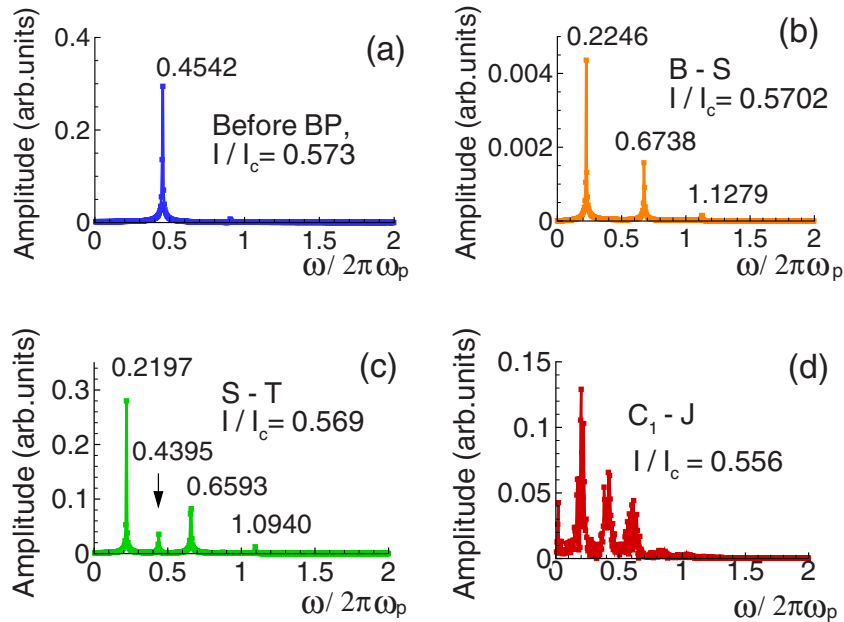

FIG. 4. (Color online) Results of FFT analysis of time dependence of the charge in the third $S$ layer in the different parts of BPR.

charge oscillations in $T-Y$ part of the BPR is different from that in the $S-T$ part of the BPR. The charge oscillations are characterized here by the beating process. A specific feature of these part is the absence of the charge oscillations in the fourth layer. At point $Y$ the charge oscillations in layer 4 grow rapidly and are getting the same order as the values in the other layers. Due to the coupling between junctions the oscillations in the other layers are increasing too, for example, similar to the third layer shown in Fig. 2(b). The $Y$ $-C_{1}$ part of the BPR [Fig. 5(c)] is characterized by the beating process as well but with an additional frequency in compare with the $T-Y$ region. The $C_{1}-J$ part of the BPR presented in Fig. 5(d) display a behavior close to the chaotic one. So, the different character of the charge oscillation in the $S$ layers is in correlation with the features of the CVC and reflects its nonlinear behavior in the BPR.

The question concerning the origin of the BPR and the fine structure in the CVC might be simplified if we take into account the results obtained for stacks with even number of IJJ in Ref. 3. In that case the LPW with wavelength $\lambda=2 d$ $(k=\pi)$ is created. This wavelength of the LPW is commensurable with the length of the stack in the $c$ direction and the BPR in the CVC does not appear. For such stacks with even number of junctions the resonance region is observed only, which corresponds to the continuous increase in the amplitude of the charge oscillations and followed by jump to the another branch.

Let us now discuss shortly the experimental situation. As we mentioned in Ref. 3, the BPR exists in the numerical simulations of the other authors as well. The points such as breakpoint are presented in the experimental CVC of Refs. 9 and 10 Recently, a new detailed experiment was made, and the clear manifestation of the BPR structure was found. ${ }^{11}$ It makes promising the possibility to observe experimentally the features presented in this paper. The following conditions
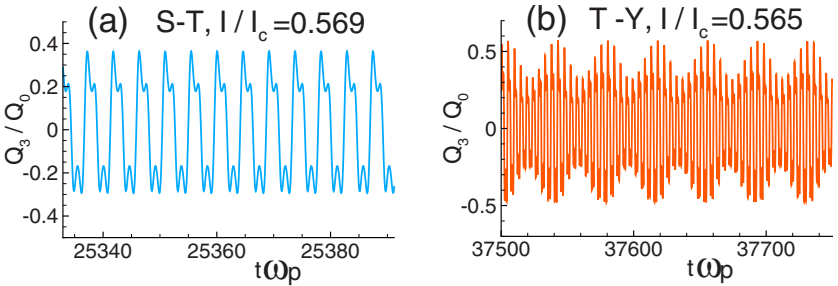

(c) $\mathrm{Y}-\mathrm{C}_{1}, \mathrm{I} / \mathrm{I}_{\mathrm{c}}=0.5625$
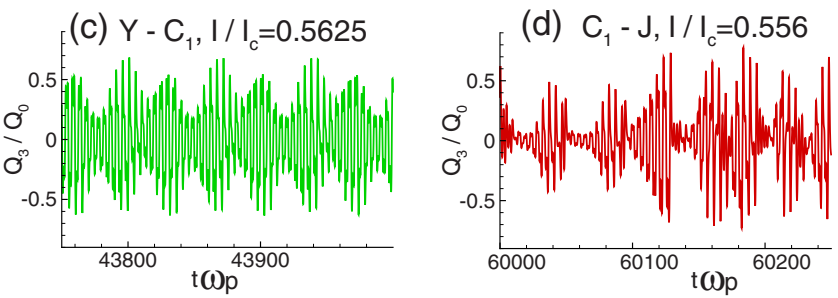

FIG. 5. (Color online) Charge oscillations in the third $S$ layer in the stack with nine IJJ in different parts of the BPR specified by the value of $I / I_{c}$

are important for that. The measurement should be done with high precision in bias current and slow sweeping of the CVC. Because the BPR region width is decreasing rapidly with the number of junctions, ${ }^{3}$ it should not be too big to observe the BPR structure. If the step in the bias current is big enough, it makes possible to pass the bifurcation points ${ }^{12}$ related to the parametric resonance in the system. The influence of the inductive coupling on the BP and BPR is not investigated yet, but if the stack's size is smaller than the Josephson penetration depth, we may neglect it.

In summary, we predict a fine structure in the outermost branch of the current-voltage characteristics in the layered superconductors. We establish a correspondence between the features of this structure and the character of the charge oscillations in the different superconducting layers in the stack. We demonstrate that the different parts of the BPR correspond to the different dynamics of the charge oscillation in the superconducting layers. Using an idea about parametric resonance at the breakpoint, we explain the origin of the BPR structure. A plasma mode is a stable solution of the system in the BPR, and this fact might be used in some applications, particularly, in high-frequency devices such as terahertz oscillators and mixers. We consider as well that the breakpoint phenomenon might be used to understand the mechanism of the powerful coherent radiation from the stack of the IJJ in BSCCO.

We thank T. Hikihara, I. Kakeya, A. Irie, H. J. Lee, K. Kadowaki, N. F. Pedersen, P. Seidel, T. Koyama, M. Machida, T. Hatano, and N. Plakida for helpful discussions. Yu.M.S. thanks J. Ishikawa and S. Noda for their kind hospitality during his stay at Kyoto University. This research was supported by Russian Foundation for Basic Research under Grant No. 08-02-00520-a. 
${ }^{1}$ L. Ozyuzer, A. E. Koshelev, C. Kurter, N. Gopalsami, Q. Li, M. Tachiki, K. Kadowaki, T. Yamamoto, H. Minami, H. Yamaguchi, T. Tachiki, K. E. Gray, W.-K. Kwok, and U. Welp, Science 318, 1291 (2007).

${ }^{2}$ Yu. M. Shukrinov and F. Mahfouzi, Supercond. Sci. Technol. 20, S38 (2007).

${ }^{3}$ Yu. M. Shukrinov, F. Mahfouzi, and N. F. Pedersen, Phys. Rev. B 75, 104508 (2007).

${ }^{4}$ Yu. M. Shukrinov and F. Mahfouzi, Phys. Rev. Lett. 98, 157001 (2007).

${ }^{5}$ M. Machida, T. Koyama, A. Tanaka, and M. Tachiki, Physica C 330, 85 (2000).

${ }^{6}$ Yu. M. Shukrinov, F. Mahfouzi, and P. Seidel, Physica C 449, 62
(2006).

${ }^{7}$ H. Matsumoto, S. Sakamoto, F. Wajima, T. Koyama, and M. Machida, Phys. Rev. B 60, 3666 (1999).

${ }^{8}$ T. Koyama and M. Tachiki, Phys. Rev. B 54, 16183 (1996).

${ }^{9}$ H. Ishida, K. Okanoue, and K. Hamasaki, Appl. Phys. Lett. 86, 122503 (2005); K. Okanoue and K. Hamasaki, ibid. 87, 252506 (2005).

${ }^{10}$ K. Tanabe, Y. Hidaka, S. Karimoto, and M. Suzuki, Phys. Rev. B 53, 9348 (1996).

${ }^{11}$ A. Irie, Yu. M. Shukrinov, and G. Oya, arXiv:0808.3896 (unpublished).

${ }^{12}$ M. Machida, T. Koyama, and M. Tachiki, Phys. Rev. Lett. 83, 4618 (1999). 